\title{
Duration after Malayan Pit Viper Bite to Detect Coagulopathy in Songklanagarind Hospital
}

\author{
Kanthika Kraisawat, M.D., Nattaya Promwang, M.D.
}

Department of Emergency Medicine, Faculty of Medicine, Prince of Songkla University, Hat Yai, Songkhla 90110, Thailand.

Received 16 November $2019 \bullet$ Revised 22 January 2020 • Accepted 27 January $2020 \bullet$ Published online 9 March 2020

\section{Abstract:}

Objective: The main objective was to determine the duration from the bite of a Malayan pit viper (MPV) until a prolonged clotting time in order to create a practice protocol for the Emergency Department (ED) of a university hospital in southern Thailand. The secondary objective was to know the incidence of adverse reactions from the MPV antivenom.

Material and Methods: This was a retrospective descriptive study of patients with MPV bite from 1 January 2006 to 30 November 2017 in the ED at Songklanagarind Hospital.

Results: The study included 153 patients. The average age was 45 years. The median elapsed time from bite to the ED was 40 minutes (interquartile range 30, 80). Fifty nine patients (38.6\%) had coagulopathy. The median elapsed time from bite to coagulopathy was 5 hours 30 minutes (range, 40 minutes to 48 hours 40 minutes). Four patients (6.8\%) had coagulopathy within the first 2 hours. Thirty patients (50.8\%) had coagulopathy within the first 6 hours. Coagulopathy was detected in most of the patients (83.1\%) within the first 24 hours. Fifty patients (84.8\%) received antivenom. Four patients had immediate hypersensitivity reactions.

Conclusion: The ED protocol should follow-up with a 20-minute whole blood clotting test at 6 hours after a bite to determine the appropriate disposition. If the patients do not have coagulopathy after 6 hours from snake bite, it is safe to follow-up at 24 hours, 48 hours and 72 hours after the bite. The incidence of antivenom hypersensitivity was low.

Keywords: coagulopathy, Malayan pit viper, venous clotting time, 20-minute whole blood clotting test

Contact: Kanthika Kraisawat, M.D.

Department of Emergency Medicine, Faculty of Medicine,

Prince of Songkla University, Hat Yai, Songkhla 90110, Thailand.

E-mail: getjung004@ hotmail.com

๑ 2020 JHSMR. Hosting by Prince of Songkla University. All rights reserved.

This is an open access article under the CC BY-NC-ND license

(http://www.jhsmr.org/index.php/jhsmr/about/editorialPolicies\#openAccessPolicy).
J Health Sci Med Res 2020;38(2):93-101 doi: $10.31584 /$ jhsmr.2020728 www.jhsmr.org 


\section{Introduction}

Venomous snakebite is an important public health problem in Thailand. Due to the topography and climate, various kinds of venomous snakes are dispersed around the region, especially in the countryside. The information from the Bureau of Epidemiology of the Ministry of Public Health shows that around 6,155 peoples were bitten annually by venomous snakes in thailand. The rate of venomous snakebite in the year 2006 was 13.25 times per 100,000 people but it decreased significantly to 1.88 times in the year 2014. Nevertheless, it increased to 7.06 times in the year 2015. ${ }^{1}$ A 3-year epidemiology study by Butaraj et al. ${ }^{2}$, based in Songkhla province, found that around 43 snakebite victims from 100,000 patients; by counting the 800,000 patients at the hospitals. They found that the snakes most likely to bite humans are Malayan pit viper (MPV) (Calloselasma rhodostoma) (94.5\%) and Cobra (4.5\%). Songklanagarind Hospital is located in Songkhla province, in southern Thailand. A MPV is the most common venomous snake found in this region, and the MPV bite is an important public health issue..$^{2-10}$ The MPV has a triangular head, with a snout turned up at the tip, a thick body with a short tail and a total length of up to one meter. Its dorsal surface has dark triangular markings, on a reddish-brown background. ${ }^{6,9}$ The venom of MPV exhibit both biological and enzymatic activities on hemostasis that include; increase vascular permeability, induced tissue necrosis, thrombin-like action, fibrinogenolytic and fibrinolytic activities, induced platelet-aggregating, inhibited platelet-aggregating and direct poisoning to platelets. Clinical manifestations of patients with MPV bites show local effects, that include swelling, pain, blister, hemorrhagic bleb, local ecchymosis and tissue necrosis. Systemic envenomation is found in $30.0-50.0 \%$ of bitten patients which causes prolonged venous clotting time (VCT) due to defibrination, low fribrinogen level, bleeding problems and thrombocytopenia. ${ }^{9}$
Following the Thai Practice Guideline for Management of Patients with Snake Bites from the Ministry of Public Health $^{3}$, the diagnosis of a victim bitten by a MPV requires evidences such as the snake carcass, lesions of the fang marks, signs, symptoms, and laboratory tests. For patients who have physical signs of a hemotoxin, the snake's venom in the blood stream will cause the important abnormal laboratory results. The VCT or the 20-minute whole blood clotting test (20WBCT) will be prolonged along with a low platelet count in serious cases.

Administration of MPV antivenom is considered when there is evidence of a bleeding disorder from a prolonged $\mathrm{VCT}^{3}$ or positive $20 \mathrm{WBCT}, 3,4,8$ or the platelet count is lower than $10 \times 10^{9}$ Liter $^{3}$, or the international normalized ratio based on the plasma prothrombin time coagulation test is more than 1.2 , or there is severe pain with swelling with suspected of compartment syndrome. ${ }^{4}$ If the VCT is longer than 20 minutes, or the 20WBCT has not clotted, the patient must be admitted or transferred to a hospital that has the antivenom. However, if the VCT or 2OWBCT is normal, the attending physicians can observe in the emergency department (ED) for around 2 hours, and repeat the VCT or 20WBCT. If it is still normal, the patient can be discharged with a follow-up VCT or 20WBCT at 24 hours and 48 hours. The patient is advised to return if a bleeding disorder occurs or a limb swells. The VCT or 20WBCT should be done every 6 hours in the noncoagulopathy inpatient. ${ }^{3}$ Five vials of MPV antivenom is administered in the coagulopathy patients having clinical of bleeding and three vials for those who do not have clinical bleeding. The VCT or 20WBCT must be followed up every 6 hours after antivenom administration until 24 hours after no systemic hemotoxic envenomation. ${ }^{3}$ If a patient has a prolonged VCT or positive 20WBCT, an additional 3 or 5 vials of antivenom is administered.

The present study aimed to analyze elapsed time from the MPV bite to a prolonged VCT or positive 20WBCT 
as information to determine the proper time to take a blood sample in patients with a MPV bite and to know whether 6 hours after the bite is proper time to detect a prolonged clotting time. The secondary objective was to detect adverse reactions for the safe use of the antivenom protocol.

\section{Material and Methods}

This was an observational, retrospective data collection study from 1 January 2006 to 30 November 2017 in patients assigned to X20 in the ICD-10 and diagnosed as Malayan pit viper bite in the outpatient department (OPD) card details. Songklanagarind Hospital is an urban teaching hospital that has an ED volume of over 48,000 patient visits per year, and has a capacity of 700 beds. A computer-based randomization system selected 427 patients who were assigned to $\mathrm{X} 20$ series. On the OPD card details, the patients who were bitten by other snakes were excluded. The number of patients enrolled into the study was 153 patients. Patients were included if the snake was captured, or the patient presented with a good description and the clinical and laboratory results were consistent with MPV envenomation. Ethics approval was obtained from the Institutional Ethics Committee Board of the Faculty of Medicine at Prince of Songkla University, project number 60407204. This study recorded the information of patients who were bitten by MPV and collected the patient characteristics that included place, site, date and time of bite, clinical features of snake envenomation, laboratory results, use of antivenom, length of hospital stay, and outcome.

The 20WBCT and VCT are very useful and informative bedside tests. The 20WBCT requires 2 milliliter $(\mathrm{mL})$ of fresh venous blood in a new, clean, dry, ordinary glass tube, and is maintained, undisturbed, for 20 minutes at an ambient temperature. The tube is then tipped to determine whether clotting has occurred. If the blood has not clotted, the patient is assumed to have systemic hemotoxic envenomation from severe hypofibrinogenemia. ${ }^{4}$
VCT requires $3 \mathrm{~mL}$ of fresh venous blood, of which $1 \mathrm{~mL}$ of blood is delivered from the plastic syringe into three glass tubes. The test tubes must be left undisturbed for 5 minutes at an ambient temperature. Then the first tube is tilted gently to check for clot formations every 30 seconds, while the other tubes are left undisturbed. After the blood in the first tube has clotted, the time is noted, then the second tube is tilted every 30 seconds and examined. Following the clotting of the second tube, the third tube is examined. The time at which clotting is observed in the third tube is the recorded time for VCT. ${ }^{11}$

\section{Statistical analyses}

Statistical analysis was conducted using $\mathrm{R}$ software version 3.2.2. Continuous variables were analyzed and are reported as mean and median, while categorical variables are reported as percentage. The student t-test was used for continuous variables, and continuous variables were compared using the Mann-Whitney U-test. Categorical variables were compared using the Pearson's chi-squared test or Fisher's exact test, as indicated. A p-value $<0.05$ was considered statistically significant. The number of prolonged VCT or positive 20WBCT patients was analyzed using the Kaplan Meier curve.

\section{Results}

\section{Demographic data}

A total of 153 patients were included in this study: 103 men (67.3\%) and 50 women (32.7\%). The average age was 45 years. The peak age range was $46-60$ years that included 46 patients $(30.0 \%)$, followed by 37 patients $(24.2 \%)$ who were over 60 years. The median elapsed time from bite to the ED was 40 minutes [interquartile range (IQR) 30, 80)]. The main site of bite injury was the lower extremities in 94 patients (61.4\%). Most of the bites occurred from 6:00 a.m. to 12:00 p.m. in 52 patients $(34.0 \%)$ and in the outdoors in 71 patients (46.4\%). Fiftynine patients $(38.6 \%)$ had coagulopathy. The median 
elapsed time from bite to coagulopathy was 5 hours 30 minutes [range, 40 minutes to 48 hours 40 minutes (IQR $3,18)$ ]. For the patient who had a prolonged VCT of 48 hours 40 minutes, which was established after the third blood test, his elapsed time from bite to the ED was 30 minutes. The median length of hospital stay (LOS) in the normal VCT group or 20WBCT group was 2 days [range, 1-4 days (IQR 2, 2)], the maximum LOS was 4 days because the patients had cellulitis. Most of the noncoagulopathy patients were admitted to observe the clinical condition of swollen limbs and for follow-ups of the VCT or 20WBCT every 6 hours during the first 24 hours and then they were discharged. The median LOS was 4 days in the coagulopathy patients [range, 2-32 days (IQR 3, 4.5)]. One coagulopathy patient had a long LOS of 32 days because of many complications including compartment syndrome, acute kidney injury and congestive heart failure. Another coagulopathy patient had a long LOS of 26 days because of necrotizing fasciitis. Both patients had no history of allergy or medical problems. The severe complications following MPV bite were necrotizing fasciitis which required surgical intervention in one patient $(0.7 \%)$, compartment syndrome in 12 patients $(7.8 \%)$ of whom half of them required surgical intervention, such as fasciotomy, wound debridement or skin grafting (Table 1). All patients with coagulopathy and complications received MPV antivenom before surgical interventions. One patient developed gingival bleeding, coagulopathy, low platelet count and cellulitis. Most MPV bites occurred in April $(15.0 \%)$ and May (13.7\%) (Figure 1).

Table 1 Demographics and characteristics of the patients

\begin{tabular}{|c|c|c|c|c|}
\hline Characteristics & $\begin{array}{l}\text { Prolonged VCT or } \\
\text { positive } 20 W B C T \\
n=59 \\
\text { Patients }(\%)\end{array}$ & $\begin{array}{l}\text { Normal VCT or } \\
20 \text { WBCT } \\
\mathrm{n}=94 \\
\text { Patients (\%) }\end{array}$ & $\begin{array}{l}\text { Total } \\
\mathrm{n}=153 \\
\text { Patients (\%) }\end{array}$ & $p$-value \\
\hline Sex & & & & 0.543 \\
\hline Male & $38(64.4)$ & $65(69.1)$ & $103(67.3)$ & \\
\hline Female & $21(35.6)$ & $29(30.9)$ & $50(32.7)$ & \\
\hline Age group (year) & & & & 0.021 \\
\hline$<15$ & $4(6.8)$ & $5(5.3)$ & $9(5.9)$ & \\
\hline $15-30$ & $5(8.5)$ & $27(28.7)$ & $32(20.9)$ & \\
\hline $31-45$ & $10(16.9)$ & $19(20.2)$ & $29(19.0)$ & \\
\hline $46-60$ & $24(40.7)$ & $22(23.4)$ & $46(30.0)$ & \\
\hline$>60$ & $16(27.1)$ & $21(22.3)$ & $37(24.2)$ & \\
\hline Place of bite & & & & 0.157 \\
\hline Indoor & $24(40.7)$ & $32(34.0)$ & $56(36.6)$ & \\
\hline Outdoor & $30(50.8)$ & $41(43.6)$ & $71(46.4)$ & \\
\hline Unknown & $5(8.5)$ & $21(22.3)$ & $26(17.0)$ & \\
\hline Site of bite & & & & 0.097 \\
\hline Upper limbs & $24(40.7)$ & $35(37.2)$ & $59(38.6)$ & \\
\hline Lower limbs & $35(59.3)$ & $59(62.8)$ & $94(61.4)$ & \\
\hline Local signs & $57(96.6)$ & $76(80.9)$ & $133(86.9)$ & 0.005 \\
\hline Tissue necrosis & $3(5.2)$ & $0(0.0)$ & $3(0.2)$ & 0.079 \\
\hline Ecchymosis/hematoma & $5(8.6)$ & $6(7.9)$ & $11(7.2)$ & 0.001 \\
\hline Blisters & $7(12.1)$ & $7(9.2)$ & $14(9.2)$ & 0.592 \\
\hline Bleeding per wound & $13(22.4)$ & $7(9.2)$ & $20(13.1)$ & 0.034 \\
\hline Limb swelling & $55(94.8)$ & $70(92.1)$ & $125(81.7)$ & 0.731 \\
\hline
\end{tabular}


Table 1 (continued)

\begin{tabular}{|c|c|c|c|c|}
\hline Characteristics & $\begin{array}{l}\text { Prolonged VCT or } \\
\text { positive } 20 W B C T \\
n=59 \\
\text { Patients }(\%)\end{array}$ & $\begin{array}{l}\text { Normal VCT or } \\
\text { 20WBCT } \\
\mathrm{n}=94 \\
\text { Patients (\%) }\end{array}$ & $\begin{array}{l}\text { Total } \\
\mathrm{n}=153 \\
\text { Patients (\%) }\end{array}$ & p-value \\
\hline Local wound complications & $46(78.0)$ & $34(36.2)$ & $80(52.3)$ & 0.000 \\
\hline Necrotizing fasciitis & $1(1.7)$ & $0(0.0)$ & $1(0.7)$ & 0.386 \\
\hline Compartment syndrome & $10(16.9)$ & $2(2.1)$ & $12(7.8)$ & 0.001 \\
\hline Cellulitis & $35(59.3)$ & $32(34.0)$ & $67(43.8)$ & 0.002 \\
\hline Time range of bite & & & & 0.734 \\
\hline 00:01 a.m.-06:00 a.m. & $12(20.3)$ & $19(20.2)$ & $31(20.3)$ & \\
\hline 06:01 a.m.-12:00 p.m. & $23(39.0)$ & $29(30.9)$ & $52(34.0)$ & \\
\hline 12:01 p.m.-06:00 p.m. & $11(18.6)$ & $20(21.3)$ & $31(20.3)$ & \\
\hline 06:01 p.m.-12:00 a.m. & $13(22.0)$ & $26(27.7)$ & $39(25.4)$ & \\
\hline $\begin{array}{l}\text { Elapsed time from bite to th } \\
\text { median (IQR) }\end{array}$ & $57(30.0,90.0)$ & $37.5(25.8,60.0)$ & $40(30.0,80.0)$ & 0.085 \\
\hline $\begin{array}{l}\text { Length of stay (days), } \\
\text { median (IQR) }\end{array}$ & $4(3.0,4.5)$ & $2(2.0,2.0)$ & $3(2.0,4.0)$ & 0.000 \\
\hline
\end{tabular}

Data are expressed as $\mathrm{n}(\%)$ unless indicated otherwise.

VCT=venous clotting time, 20WBCT=20-minute whole blood clotting test, IQR=interquartile range, ED=emergency department

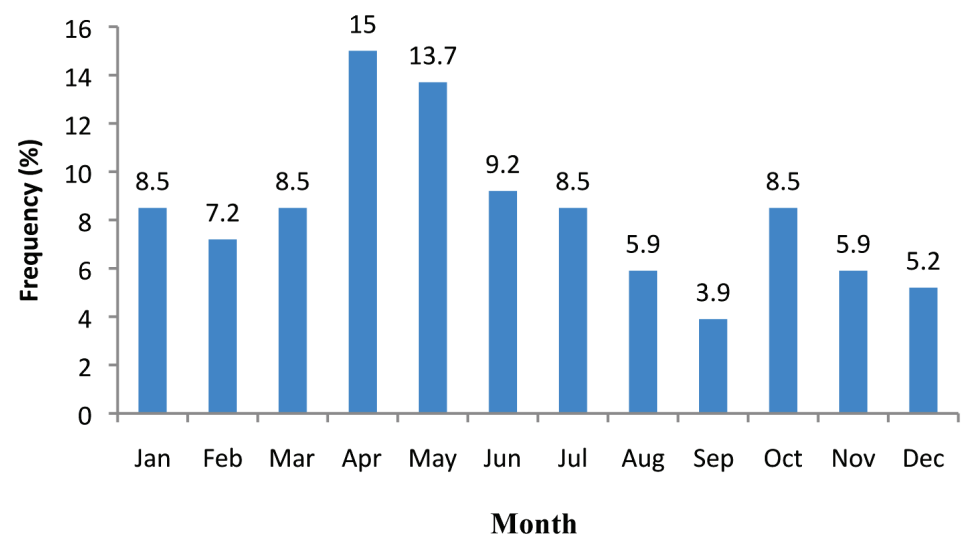

Figure 1 Seasonal frequency of Malayan pit viper bites (\%) by month from January 2006 to November 2017

Four patients $(6.8 \%)$ were detected coagulopathy within the first 2 hours, and in half of all patients $(50.9 \%)$ coagulopathy was detected within the first 6 hours. Coagulopathy was detected in most of the patients (83.0\%) within the first 24 hours (Figure 2). 


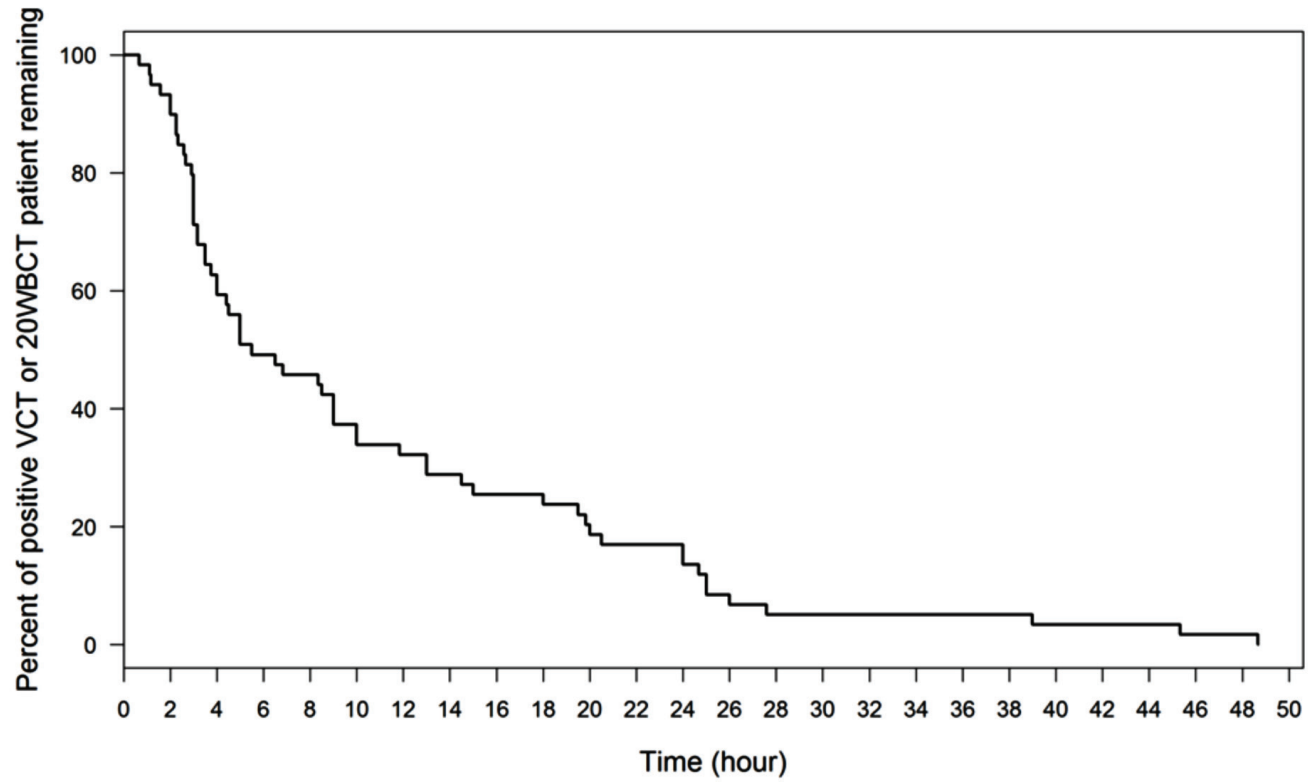

VCT=venous clotting time, $20 \mathrm{WBCT}=20$-minute whole blood clotting test

Figure 2 Modified Kaplan Meier Curve of prolonged venous clotting time, or positive 20 minute whole blood clotting test patients $(n=59)$

Fifty patients $(84.7 \%)$ were treated with antivenom in Songklanagarind Hospital. Nine patients (15.3\%) were transferred to receive antivenom at a nearby hospital. Forty-six patients (92.0\%) received monovalent MPV antivenom and 4 patients $(8.0 \%)$ received polyvalent hemotoxin antivenom. Only one patient received premedication because of asthma. Four patients exhibited immediate hypersensitivity reactions, following administration of intravenous antivenom. Three patients developed bronchospasm and four patients had a rash. None of the patients with immediate hypersensitivity reactions had received pre-medication prior to antivenom administration.

Most of the coagulopathy patients $(70.0 \%)$ required 3 to 6 vials of antivenom. The median number of antivenom vials was 5 [range, 3-30 vials (IQR 3; 9.25)] (Table 2). Many patients received more than 5 vials of antivenom, because the VCT was still prolonged after the first or second administration of antivenom.
Table 2 Number of antivenom administration $(n=50)$

\begin{tabular}{ll}
\hline Number of antivenom (vials) & Number of patients (\%) \\
\hline $3-6$ & $35(70.0)$ \\
$7-12$ & $5(10.0)$ \\
$13-18$ & $8(16.0)$ \\
$19-24$ & $1(2.0)$ \\
$\geq 25$ & $1(2.0)$ \\
\hline
\end{tabular}

\section{Discussion}

Although the city of Hat Yai is a developed city in Songkhla province, the MPV is still common because the surrounding countryside has rubber plantations. The peak incidence is during the morning hours between 6:00 a.m. and 12:00 p.m. and the peak season is summer, which corresponds with the time the patients worked in fields or rubber plantations. The most common bite site is the lower extremities according to Laohawiriyakamol et al. ${ }^{5}$ and Wongthongkam et al. $^{6}$ studies. The most common 
degree of severity is mild severity such as local effects with mild swelling. The median elapsed time from bite to the ED was 40 minutes. Therefore, most of the patients arrived early at the ED. The present study did not differ from the Deechaisest ${ }^{7}$ study that found that the most common elapsed time from bite to the ED was within 60 minutes. The Wongthongkam et al. ${ }^{6}$ study had a longer mean elapsed time from bite to the ED (175 minutes) because most of the bites occurred in rural areas.

The VCT has been performed by the Songklanagarind Hospital physicians during previous years. However the World Health Organization ${ }^{8}$ and other international institutions have promoted the 20WBCT for patient evaluation. During the last three years, the Songklanagarind Hospital physicians prefer performing the 20WBCT. The present study found that 59 patients (38.6\%) with MPV bite had a prolonged VCT or positive 20WBCT. These results did not differ greatly from the Lekhakula $^{9}$ study (30.0\%) and the Wongthongkam et al. ${ }^{6}$ study (35.4-52.5\%). The incidence of coagulopathy was lower in this current study than the Deechaisest ${ }^{7}$ study $(59.3 \%)$.

The median elapsed time from the MPV bite to the prolonged clotting time was 5 hours 30 minutes [range, 40 minutes to 48 hours 40 minutes (IQR 3, 18)]; however, the results were 1 hour to 72 hours in the Deechaisest ${ }^{7}$ study. The study by Warrell et al. ${ }^{10}$ found that non-clotting blood was from 1.5 to 72 hours (mean, 27.5 hours). The study by Wongthongkam ${ }^{6}$ found that coagulopathies appeared within the first 24 hours. Half of the coagulopathy patients $(50.8 \%)$ were detected within 6 hours after the MPV bite. Coagulopathies occurred within the first 24 hours in $83.1 \%$ of patients, whereas the Deechaisest ${ }^{7}$ study reported that coagulopathy occurred within the first 24 hours in $93.8 \%$ of patients. The present study found that coagulopathy occurred within the first 2 hours in $6.8 \%$ of patients and within the first 6 hours in $50.8 \%$ of patients. However, the Deechaisest ${ }^{7}$ study found that positive results occurred within the first 6 hours in $31.3 \%$ of patients. Therefore, it may be safe to follow-up the VCT or 20WBCT as an inpatient within the first 24 hours. Since coagulopathy occurred in $83.1 \%$ during the first 24 hours, the attending physician should admit all patients with MPV bite to perform the 20WBCT every 6 hours within the first 24 hours after the bite, if possible. The clinical conditions of patients with MPV bite should be observed and followed up with the VCT or 20WBCT at 6 hours after the bites to determine an appropriate disposition. The non-coagulopathy patients can be discharged with a follow-up VCT or 20WBCT at 24 hours, 48 hours, and 72 hours after being bitten.

The antivenoms were lyophilized equine $F(a b)_{2}$ species-specific antibody produced by Queen Saovabha Memorial Institute (QSMI), Bangkok, Thailand. The present study reported a low incidence of antivenom hypersensitivity (8.0\% or 4 patients), which was lower than the Blessmann et al. ${ }^{12}$ study $(17.4 \%$ or $4 / 23$ patients who received MPV antivenom) and the Deechaisest ${ }^{7}$ study (12.5\% or 2 patients who developed rash and no anaphylaxis occurred), but higher than the Thiansookon et al. ${ }^{13}$ study (1.6\% or $4 / 254$ patients) and the Wongthongkam ${ }^{6}$ study $(1.8 \%$ or 2 patients who developed mild to moderate serum sickness and no case of anaphylaxis). All studies used the same antivenom from QSMI. A further study should focus on pre-medication before antivenom administration and the correlation with clinical outcomes. The present study found that the median LOS of the coagulopathy patient was 4 days [range, 2-32 days (IQR 3; 4.5)], which was similar to the LOS results in Deechaisest ${ }^{7}$ study (range, 1-4 days), and the Wongthongkam et al. ${ }^{6}$ study (LOS, 3-5 days). The present study found that the patients with prolonged LOS had local wound compli- 
cations and required surgical intervention. One patient had necrotizing fasciitis $(0.7 \%), 12$ patients had compartment syndrome $(7.8 \%)$, and seven patients $(4.6 \%)$ required surgical interventions. According to the Wongthongkam et al. $^{6}$ study, $4.0 \%$ of cases (9/225 patients) required surgical interventions.

\section{Limitations}

The present study was a retrospective study. The authors did not obtain the eventual clinical outcomes of the transferred patients, or the long-term clinical outcomes after discharge. The accuracy of elapsed time from bite to coagulopathy was limited, because coagulopathy actually became abnormal before the follow-up times. A prospective study should be conducted in the future.

\section{Conclusion}

Detecting coagulopathy within the first 2 hours after MPV bite may cause a low detection rate of coagulopathy patients. The clinical conditions of the patient should be observed and the 20WBCT should be performed at 6 hours after the bite before determining an appropriate disposition. If the patients do not have coagulopathy after 6 hours of a snake bite, it is safe to follow-up at 24 hours, 48 hours and 72 hours after the bite. The incidence of antivenom hypersensitivity was low; however, severe, immediate hypersensitivity reactions could occur.

\section{Acknowledgement}

The authors thank Kingkarn Waiyanak for article searches and retrieval, Glenn K. Shingledecker and Rapeepun Saiutus for thier help in editing the manuscript.

\section{Funding sources}

Faculty of Medicine, Prince of Songkla University

\section{Conflict of interest}

None

\section{References}

1. Muang Dan C. Snake bite. In: Siripanich S, editor. Annual epidemiological surveillance report 2015. Nonthaburi: Bureau of Epidermiology, National Trustworthy and Competent Authority in Epidemiological Surveillance and Investigation; 2015;p.178.

2. Butaraj S. Epidemiological study of venonous snakes in Songkhla province. J Commun Dis 1984;10:337-50.

3. The academic development of Department of Medical Services, The Ministry of Public Health. Practice guideline for management of patients with snake bite. Bangkok: The Agricultural Cooperative Federation of Thailand; 2004.

4. Regional Office for South-East Asia, World Health Organization. Guidelines for the management of snakebites. $2^{\text {nd }} \mathrm{ed}$. New Delhi: WHO Regional Office for Southeast Asia; 2016.

5. Laohawiriyakamol S, Sangkhathat $S$, Chiengkriwate $P$, Patrapinyokul $S$. Surgery in management of snake envenomation in children. World J Pediatr 2011;7:361-4.

6. Wongthongkam N, Wilde H, Sitti-Amorn C, Ratanabananangkoon K. A study of 225 Malayan pit viper bites in Thailand. Mil Med 2005;170:342-8.

7. Deechaisest P. The outcome of Malayan pit viper (Calloselasma rhodostoma) antivenom in snake bite victims at $\mathrm{Na}$ Mom Hospital, Songkhla province, Thailand. J Health Sci 2012;21: 1157-65.

8. Warrell DA. Guideline for management of snakebite. New Delhi: WHO Regional Office for Southeast Asia; 2010.

9. Lekhakula A. Management of Malayan pit viper bites. J Hematol Transfus Med 2014;24:163-73.

10. Warrell DA, Looareesuwan S, Theakston RD. Randomized comparative trial of three mono specific antivenoms for bites by Malayan pit viper (Colloselasma rhodostoma) in southern Thailand: clinical and laboratory correlations. Am J Trop Med Hyg 1986;35:1235-47.

11. Lee RI, White PD, A clinical study of the coagulation time of blood. Am J Med Sci 1913;145:496-503.

12. Blessmann J, Khonesavanh C, Outhaithit P, Manichanh S, Somphanthabansouk K, Siboualipha P. Venomous snake 
bites in Lao PDR: a retrospective study of 21 snakebite victims in a provincial hospital. Southeast Asian J Trop Med Public Health 2010;41:195-202.
13. Thiansookon A, Rojnuckarin P. Low incidence of early reactions to horse-derived $F\left(a b^{\prime}\right)(2)$ antivenom for snakebites in Thailand. Acta Trop 2008;105:203-5. 